\title{
Broad-spectrum Non-toxic Antiviral Nanoparticles with a Virucidal Inhibition
}

\section{Mechanism}

Valeria Cagno ${ }^{1,2,3} \dagger$, Patrizia Andreozzi ${ }^{4,5} \dagger$, Marco D’Alicarnasso ${ }^{6}$, Paulo Jacob Silva ${ }^{2}$, Marie Mueller $^{2}$, Marie Galloux ${ }^{7}$, Ronan Le Goffic ${ }^{7}$, Samuel T. Jones ${ }^{2,8}$, Marta Vallino ${ }^{9}$, Jan Hodek ${ }^{10}$, Jan Weber ${ }^{10}$, Soumyo Sen ${ }^{11}$ Emma-Rose Janeček ${ }^{2}$, Ahmet Bekdemir ${ }^{2}$, Barbara Sanavio ${ }^{12}$, Chiara Martinelli ${ }^{4}$, Manuela Donalisio ${ }^{1}$, Marie-Anne Rameix Welti ${ }^{13,14}$, Jean-Francois Eleouet ${ }^{7}$ Yanxiao Han $^{11}$, Laurent Kaiser ${ }^{15}$, Lela Vukovic ${ }^{16}$, Caroline Tapparel ${ }^{3,15}$, Petr Král ${ }^{11,17}$, Silke Krol ${ }^{12,18}$, David Lembo ${ }^{1 *}$, Francesco Stellacci ${ }^{2,19 *}$

${ }^{1}$ Dipartimento di Scienze Cliniche e Biologiche, Univerisita' degli Studi di Torino, Orbassano, Italy.

${ }^{2}$ Institute of Materials, Ecole Polytechnique Fédérale de Lausanne (EPFL), Lausanne, Switzerland.

${ }^{3}$ Faculty of Medicine of Geneva, Department of Microbiology and Molecular medicine, Geneva, Switzerland.

${ }^{4}$ IFOM - FIRC Institute of Molecular Oncology, IFOM-IEO Campus, Milan, Italy.

${ }^{5}$ CIC biomaGUNE Soft Matter Nanotechnology Group San Sebastian-Donostia, Spain.

${ }^{6}$ Fondazione Centro Europeo Nanomedicina (CEN), Milan, Italy.

${ }^{7}$ VIM, INRA, Université Paris-Saclay, Jouy-en-Josas, France.

${ }^{8}$ Jones Lab, School of Materials, University of Manchester, Oxford Road, Manchester, M13 9PL

${ }^{9}$ Istituto per la Protezione Sostenibile delle Piante, CNR, Torino, Italy.

${ }^{10}$ Institute of Organic Chemistry and Biochemistry, Academy of Science of the Czech Republic, Prague, Czech Republic.

${ }^{11}$ Department of Chemistry, University of Illinois at Chicago, USA.

${ }^{12}$ Fondazione IRCCS Istituto Neurologico «Carlo Besta», IFOM-IEO Campus, Milan, Italy.

${ }^{13}$ UMR INSERM U1173 I2, UFR des Sciences de la Santé Simone Veil—UVSQ, Montigny-LeBretonneux, France

${ }^{14}$ AP-HP, Laboratoire de Microbiologie, Hôpital Ambroise Paré, 92104, Boulogne-Billancourt, France

${ }^{15}$ Geneva University Hospitals, Infectious Diseases Divisions, Geneva, Switzerland. 
${ }^{16}$ Department of Chemistry, University of Texas at El Paso, USA.

${ }^{17}$ Department of Physics and Department of Biopharmaceutical Sciences, University of Illinois at Chicago, USA.

${ }^{18}$ IRCCS Istituto Tumori "Giovanni Paolo II", Bari, Italy

${ }^{19}$ Interfaculty Bioengineering Institute, Ecole Polytechnique Fédérale de Lausanne (EPFL), Lausanne, Switzerland. 
ABSTRACT

Viral infections kill millions yearly. Available antiviral drugs are virus-specific and active against a limited panel of human pathogens. There are broad-spectrum substances that prevent the first step of virus-cell interaction by mimicking heparan-sulfate proteoglycans (HSPG), the highlyconserved target of viral attachment ligands (VAL). The reversible binding mechanism prevents their use as a drug, because, upon dilution, the inhibition is lost. Known VAL are made of closely packed repeating units but the aforementioned substances are able to bind only a few of them. We designed antiviral nanoparticles with long and flexible linkers mimicking HSPG, allowing for effective viral association with a binding that we simulate to be strong and multivalent to the VAL repeating units, that generates forces $(\sim 190 \mathrm{pN})$ that eventually lead to irreversible viral deformation. Virucidal assays, electron microscopy images, and molecular dynamics simulations support the proposed mechanism. These particles show no cytotoxicity, and in vitro nanomolar irreversible activity against Herpes Simplex Virus (HSV), Human Papilloma Virus, Respiratory Syncytial Virus (RSV), Dengue and Lenti virus. They are active ex vivo in human cervicovaginal histocultures infected by HSV-2 and in vivo in mice infected with RSV. 
Infectious diseases account for $\sim 20 \%$ of global mortality, and viruses are responsible for about one third of these deaths. ${ }^{1}$ Lower respiratory infections and Human Immunodeficiency Virus (HIV) are among the first ten causes of deaths worldwide, and they contribute substantially to healthcare costs. ${ }^{2}$ Emerging viruses (e.g. Ebola) add yearly to this death toll. The best approach to prevent viral infections is vaccination, however there exist only a limited number of vaccines and the ones that exist are not equally available in all parts of the world. ${ }^{3}$ After infection, antiviral drugs are the only treatment option, but even in this case there are only a limited number of approved antiviral drugs and they are all virus specific. There is a dire need for broad-spectrum antiviral drugs that can act on a large number of existing and emerging viruses.

Current therapeutics can be subdivided into i) small molecules (e.g. nucleoside analogues and peptidomimetics), ii) proteins able to stimulate the immune response (e.g. interferon), and iii) oligonucleotides (e.g. fomivirsen). ${ }^{4}$ They are mainly directed against HIV, hepatitis B virus (HBV), hepatitis C virus (HCV), herpes simplex virus (HSV), human cytomegalovirus (HCMV) and influenza virus. They act intracellularly, mostly on viral enzymes that are essential for viral replication but differ from any other host enzyme to allow for selectivity. Since viruses largely depend on the biosynthetic machinery of infected cells for their replication the specificity of antiviral drugs is far from ideal, resulting in a general intrinsic toxicity associated with such treatment. ${ }^{5,6}$ Additionally, most viruses mutate rapidly due to error-prone replication machinery, therefore they often develop resistance. ${ }^{7}$ Finally, the use of virus specific proteins as a target of antiviral drugs makes it difficult to develop broad-spectrum antivirals capable of acting on a large number of viruses that are phylogenetically unrelated and structurally different.

Virustatic substances act outside the cell by interfering with the first phases of the viral replication cycle. They can be broad spectrum and non-toxic. Their activity depends on a reversible 
binding event; the reversibility of the mechanism makes them medically irrelevant. For example, upon dilution the substance is detached from an unaltered viral particle allowing the virus to infect again. To achieve broad-spectrum efficacy, current virustatic materials (e.g. heparin, polyanions) target virus-cell interactions that are common to many viruses. One of these interactions is that between the VAL and its associated cell receptor responsible for the first step of the virus replication cycle. Many viruses, including HIV-1, HSV, HCMV, HPV, RSV and Flavivirus, ${ }^{8}$ exploit HSPGs as the target of their VALs. HSPGs are expressed on the surface of almost all eukaryotic cell types. The binding between viruses and HSPGs usually occurs via the interaction of closely-packed arrangements of multiple basic amino acids on the proteins, that constitute the VAL, with the negatively charged sulfated groups of heparan sulfate (HS) in the glycocalix of the cell surface. ${ }^{9}$ A long list of HSPG mimicking materials such as heparin, ${ }^{10,11}$ sulfated polysaccharides, ${ }^{9,12}$ or sulfonic acid decorated polymers, dendrimers, and nanoparticles ${ }^{13-17}$ have been tested and shown to exert potent virustatic activity in vitro, none have shown efficacy in humans. The only three polyanionic anti-HIV-1 microbicides that reached phase III clinical trial (i.e. polysulfonated PRO2000, the polysulfated Carraguard, and cellulose sulfate Ushercell) did not prevent vaginal HIV-1 transmission and in some cases even increased the rate of infection. ${ }^{18-}$ ${ }^{21}$ One of the possible explanations is that their effect was simply virustatic and hence vaginal and seminal fluids lead to the dilution of both the viruses and the active compounds, which resulted in the complete loss of binding and release of active virus.

Arguably, the ideal drug against a viral infection would be virucidal. Virucidal molecules cause irreversible viral deactivation, indeed their effect is retained even if dilution occurs after the initial interaction with the virus. ${ }^{22}$ There is a vast literature on many virucidal materials ranging from simple detergents, to strong acids, or more refined polymers, ${ }^{23}$ and nanoparticles (NPs) ${ }^{24-27}$ 
that, in some cases, are capable of releasing ions. ${ }^{28,29}$ In all cases, the approaches utilized have intrinsic cellular toxicity. ${ }^{26}$ Indeed, all of these materials attempt to chemically damage the virus, but it is a tall order to selectively damage a virus without affecting the host the virus replicates within.

An ideal drug should have all the positive properties of virustatic drugs such as broadspectrum efficacy and low toxicity, and at the same time show a virucidal mechanism. In this paper, we show that it is possible to change the mechanism of inhibition of an antiviral nanoparticle from virustatic to virucidal by engineering its linkers in a way that we hypothesize leads to multivalent binding (i.e. the binding of multiple targets at the same time) with the consequent generation of irreversible local distortion as schematically illustrated in Figure 1A. Most VALs have binding domains composed of closely packed repeating units, hence they are ideally suited for multivalent binding to their cell receptor. All the known HSPG-mimicking NPs, polymers and dendrimers ${ }^{15}$ display short linkers to expose sulfonate groups to the viral ligands, including gold NPs coated with 3-mercaptoethylsulfonate (MES) ${ }^{16}$ and heparin. The relative rigidity of the sulfonate linkers should reasonably lead to the binding of only a few of the repeating units that constitute a VAL. Consequently, the resulting binding is weak and reversible. ${ }^{31,32}$ On the other hand, it is known $\mathrm{n}^{31,32}$ that particles, when binding strongly to a membrane (i.e. a vesicle, but we extrapolate this also to viral envelopes or capsids), can lead to significant local distortions. Hence, we replace the short linkers in MES-NPs with long ones, to achieve strong multivalent binding. We show here that strong multivalent binding leads to local distortions and eventually to a global virus deformation, with the consequent irreversible loss of infectivity. We compare MES coated gold NPs, as well as heparin, with a series of NPs coated with undecanesulfonic acid (MUS) containing ligands. All NPs show in vitro inhibition of many HSPG dependent viruses either 
enveloped (HSV, RSV, Lentivirus and Dengue virus) or naked (HPV). But while the effect of the MES-NPs and heparin is lost with dilution, all MUS coated NPs show a clear irreversible effect. As expected the 'upgrade' from a virustatic to a virucidal mechanism adds to all of the positive traits of the former (i.e. minimal toxicity and in-vitro broad spectrum efficacy) a strong effect exvivo on human cervicovaginal histocultures infected by HSV-2 that is absent in the parental virustatic drugs and a strong effect in-vivo in mice infected with RSV.

\section{Virus and Nanoparticles Description}

To evaluate the inhibitory activity of our nanoparticles (NPs) we used the following viruses: HSV type 1 (HSV-1), HSV type 2 (HSV-2), pseudoviruses of human papillomavirus type 16 (HPV-16), RSV, vescicular stomatitis virus pseudo-typed lentivirus (LV-VSV-G) and Dengue virus. All of the viruses above are HSPG dependent viruses. We used adenovirus-5 (AD5), a nonHSPG dependent virus, as a control. To mimic HSPG, we prepared NPs coated with MES and NPs coated with MUS. MES-NPs are reported in literature and are supposed to be virustatic, i.e. the sulfonic acid moieties at the end of their short linkers are effective mimics of HSPG and as a consequence they show good efficacy against a number of HSPG-dependent viruses. The postulated mechanism of virus binding to HSPGs is reversible in nature. To render it irreversible we chose to replace MES with MUS as this ligand has a long hydrophobic backbone terminating with a sulfonic acid, allowing its terminal group to move with some freedom. Consequently, NPs coated with MUS are ideal for multivalent binding, in this case the binding of multiple sulfonic acids to the HSPG-interacting motifs on the virus surface. Gold NPs coated with MUS ligands were selected, as they are the simplest non-toxic particles that can be synthesized with these ligands. Other NPs selected in the present study are the particles coated with a 2:1 mixture of MUS 
and 1-octanethiol (OT), as they are the most biocompatible, soluble, and resistant to protein nonspecific adsorption version of MUS-coated gold particles that we have studied. ${ }^{33-36}$ All used NPs are summarized in Table 1, and all synthetic methods and characterizations are presented in the supplementary information (SI) (Methods Section and Supplementary Figures 1 to 7).

\section{Viral Inhibition}

Each virus was pre-incubated with different doses of gold NPs for $1 \mathrm{~h}$ at $37^{\circ} \mathrm{C}$ and $5 \% \mathrm{CO}_{2}$; then the mixture was added to the cell culture (see Methods Section in SI for virus-specific protocol details, initial viral load, and cell types), and infectivity was tested $24-72 \mathrm{~h}$ post infection. For the GFP expressing viruses (LV-VSV-G, AD-5 and HPV-16) the infectivity was quantified by flow cytometry, while plaque assays were used for wild-type viruses. Table 2 summarizes the results. It is noteworthy that the MUS functionalized NPs i) are indeed non-toxic at these concentrations showing favourable selectivity indexes, ii) are able to inhibit infection selectively for HSPG dependent viruses (i.e. no inhibition is observed for $\mathrm{AD5}$ ), and that iii) all $\mathrm{EC}_{50}$ are in the nanomolar range (see Methods Section in SI for calculations of moles of NP). It is important to underline that the monomeric sulfonated ligand (MUS molecule) was not effective in inhibiting LV-VSV-G (Supplementary Figure 8). One possible explanation for the lack of inhibition for the MUS molecule could be interactions between various chemical groups on the surface of viruses with the thiols at the end of the ligands. We believe that this explanation is not the correct one as, no inhibitory activity of sodium undec-10-enesulfonate (pre-MUS), a molecule equivalent to MUS but lacking the thiol end-group, was detected against all the viruses tested. 
To further test that the NPs affect infectivity by mimicking the attachment receptor for HSPG-binding viruses, we performed a series of control experiments. gold NPs coated with 11mercaptoundecylphosphoric acid (MUP) ligands (Supplementary Figure 5) were synthesized, thus creating NPs of similar size, ligand- and charge-density to the MUS-NPs but replacing the sulfonate with phosphonate groups. In contrast to the MUS-NPs, the MUP-NPs showed no inhibitory activity when mixed with pseudo-lentivirus (LV-VSV-G), highlighting the importance of the sulfonic acid group for the activity of the particles. Finally, no inhibitory activity of $15 \mathrm{~nm}$ in diameter citrate-coated gold NPs was detected. In Supplementary Discussion 1 we detail experiments aimed at establishing that the particles actually do target the HSPG seeking VAL.

\section{Virucidal Results}

As explained above, other sulfonated materials ${ }^{16-19}$ have also shown similar inhibitory effects as shown for MUS-NPs in Table 2, but these effects have been proven ${ }^{10,11}$ or are assumed 33 to be virustatic and hence due to reversible attachment alone. To test whether a different inhibition mechanism was in place for our particles, we first verified the ability of our NPs to inhibit viral attachment, as is known for heparin (Supplementary Figure 9). Then, we verified the ability of MUS:OT-NPs, MES-NPs, and heparin of inhibiting viral infection. The results are shown in the blue curves in Figure 1B and summarized in Table 1. In all cases we observed $\mathrm{EC}_{50}$ in similar ranges. The inhibition assays were completed by standard toxicity tests. The orange curves in Figure 1B illustrate the results of cell viability studies. In all three cases no toxic effect was observed even at the highest concentrations. We then tested them for irreversible inhibitory activity through virucidal assays. These assays consist of an incubation of the virus and drugs at a 
concentration corresponding to the $\mathrm{EC}_{90}$ for a given amount of time and the subsequent evaluation

of the residual infectivity of the virus through serial dilutions of the inoculum. It is known ${ }^{19}$ that if the effect is solely virustatic, the viral infectivity is fully recovered upon dilution, as we show here for heparin and MES gold NPs against HSV-2 (Figure 1B). As expected in both cases we found these particles to have inhibitory activity in the nanomolar range, ${ }^{16}$ but virucidal tests showed recovery of the viral infectivity indicating a simple virustatic inhibitory mechanism. If irreversible changes are induced in the virus particle, the infectivity is never regained at all dilutions tested, even though the dilution leads to a final concentration lower than the active dose ${ }^{22}$. MUS:OT-NPs also showed nanomolar inhibition of HSV-2 infectivity but, in contrast to heparin and MES-NPs, no infectivity was regained upon dilution (Figure 1B), confirming an irreversible effect (virucidal). In agreement with our hypothesis, all HSPG-binding viruses showed irreversible loss of infectivity when incubated with MUS:OT-NPs, although to differing extents (Figure 1C).

The HSV-2 virucidal tests were performed also at different time points, as shown in Figure 1D. While the virustatic effect is immediate, as shown by dose response curve at time $0 \mathrm{~h}$ in Supplementary Figure 10, the virucidal activity develops over time, with the effect being almost complete after 30 min. Indeed, when viruses and MUS:OT-NPs were mixed and immediately added to cells, the inhibitory potency is reduced as compared to the pre-incubation experiment, confirming the time-dependent virucidal effect (Supplementary Figure 10).

\section{NPs-induction of Irreversible Changes in the Virus Particles}

To elucidate the fate of the viruses after NPs binding we performed a series of transmission electron microscopy (TEM) studies on HSV-2 exposed to MUS:OT-NPs and MES-NPs. Dry 
uranyl acetate negatively stained TEM were complemented by cryo-TEM studies (see Supplentary Discussion 2 for the choice of imaging and its validity). Figure 2 shows negative staining TEM (A) and cryo-TEM (B and C) images of viruses with and without NPs. It is possible to see different types of NP-virus association, categorized as follows: (1) virus with no NPs associated, (2) virus with some NPs associated (with particles being mostly isolated), (3) virus with NPs associated with at least one local cluster of NPs, and (4) deformed viruses mostly covered with NPs. We believe that stage (2) indicates that NPs have associated with the HSPG VALs, as time progresses the VAL attracts more particles leading to stage (3) forming NPs clusters; stage (4) is when the particles are associated with a broken virus or break the virus. Control experiments with particles that had no sulfonic acids show mostly stage (1) and in some rare cases stage (2) that we attribute to stochastic interactions. (Supplementary Figures 11).

The quantification of Cryo-TEM images illustrated in Figure 2 (D) shows that, immediately after mixing HSV-2 with MES NPs $(0.2 \mathrm{mg} / \mathrm{ml}$, approximate incubation time of $30 \mathrm{sec}), 75 \%$ of the viruses do not show any association with the NPs (stage 1), while $25 \%$ are associated with the particles (stage 2 and 3). For stage (2) and (3) we observed association primary at a single point. After 90 min of incubation at $37^{\circ} \mathrm{C}, 5 \% \mathrm{CO}_{2}$, we find that the fractions of stage (1) versus NPassociated stages remain practically unchanged. The only noticeable difference we found is that the fraction of viruses that was previously only associated to isolated particles (stage 2) now shows predominantly clusters (stage 3), with a 5\% showing stage (4) deformed viruses fully coated with NPs. Our interpretation of this data is that in MES-NPs we observe an overall sporadic sizeable interaction with the VAL leading to a progression from stage (2) to stage (3), while the fraction in stage (4) provides us with a baseline to determine the fraction in our samples of deformed viruses that have lost their capsid and get coated with NPs. 
At the same concentration as MES-NPs, the effect of MUS:OT-NPs is markedly different. In this case, all viruses immediately associate with particles, showing $50 \%$ of stage (2) and $20 \%$ of stage (3), and already $30 \%$ of the viruses are deformed and fully coated (stage 4). After 90 min images show an evolution of the interaction, as only $13 \%$ of the viruses remain in stage (2) and the other $87 \%$ are deformed and fully coated (stage 4$)$.

In our interpretation stage (2) and (3) are the imaging of a virustatic effect as they show NPs attached to viruses, while stage (4) is related to the virucidal effect, as it images viruses fully covered with NPs that most probably have lost their structural integrity. When comparing the images for MES-NPs and viruses with those for MUS:OT-NPs and viruses it is noticeable that the immediate association suggests stronger interaction with MUS:OT-NPs as images lack stage (1). While comparison of the images obtained at $90 \mathrm{~min}$ indicate that MUS:OT-NPs induce damage to a fraction of the viruses that is significantly higher to what observed for MES-NPs (87\% vs. 5\%, respectively). Moreover, image analysis leads to the conclusion that the virucidal action of the MUS:OT-NPs is progressive with time, as established also with virucidal assays (Fig. 1D), as the fraction of viruses imaged in stage (2) and (3) progressively evolves into stage (4). A similar progression can be observed with HPV-16 (Supplementary Figure 12). See Supplementary Discussion 3 for gel studies to show that changes on the viruses happen on a whole population.

\section{Mechanistic Understanding via Simulations}

In order to understand how MUS-type NPs can induce irreversible changes upon interaction with HSPG-VAL, we performed atomistic molecular dynamics (MD) simulations of different NPs interacting with the capsid of HPV-16 (Figure 3). The simulations were performed in physiological solutions, where NPs were placed close to the solvent-exposed HSPG binding 
sites (amino acid residues K278, K356, K361, K54 and K59) ${ }^{37,38}$ at the surface of HPV-16 capsid L1 proteins.

The simulation results for MUS:OT-NP (2.4 nm core and two types of ligands, MUS and OT, 50 ligands of each kind) in Figure 3A (Movie M1) demonstrate that selective multivalent binding $^{39}$ develops between negative sulfonate groups of MUS:OT-NP and positive HSPGbinding lysine residues of L1 capsid protein complexes from the HPV-16 capsid. Within 50-80 ns, 5-6 local charge interactions form on average between NP terminal sulfonate groups and L1 HSPG-binding sites (Supplementary Figures 13 and 14), which are supported by a similar number of non-local coupling contacts between nonpolar alkyl chains of NP ligands and L1 proteins. Each of the 5-6 sulfonate groups binds to positively charged amine groups of lysine residues with a relatively large Gibbs free energy of $-6 \mathrm{kcal} / \mathrm{mol}^{40}$, totaling in $\Delta \mathrm{G}_{\mathrm{bind}} \sim-34 \mathrm{kcal} / \mathrm{mol}$, while the non-polar ligand chains acquire on average a non-local total binding energy of $-21 \mathrm{kcal} / \mathrm{mol}$ (see Methods in SI for details).

This multivalent binding can induce large stresses and deformations of the L1 complexes. Given the local nature of binding of the sulfonated groups, we can use this binding to estimate the effective force with which the NPs act on the L1 complexes. By considering the increase of binding energy, $\Delta \mathrm{G}_{\mathrm{bind}}$, during the NP motion, $\Delta \mathrm{x}$, on the capsid surface, we can get an effective force that drives the NP binding and capsid deformation process forward, $\mathrm{F} \sim-\Delta \mathrm{G}_{\mathrm{bind}} / \Delta \mathrm{x}$ (Figure $3 \mathrm{C}$ ). By combining the above Coulombic energy change of $\Delta \mathrm{G}_{\text {bind }} \sim-28 \mathrm{kcal} / \mathrm{mol}$ (considering at the beginning of simulations NP interacting with a Lysine) with a distance of $10.4 \AA$ over which the MUS:OT-NP moves (Supplementary Figure 15), while acquiring this binding energy, we obtain an effective forces of $\mathrm{F} \sim 189 \mathrm{pN}$. This force can deform the L1 complexes and even disturb a 
relative position of one L1 pentamer with respect to a neighboring L1 pentamer (Figure 3C, Supplementary Figure 16 and Movie M2). This disruption of viral capsids by NPs with a multivalent Coulombic binding is analogous to the pore formation in neural membranes by $\mathrm{Ca}^{2+}$ ions ${ }^{41}$. See Supplementary Discussion 4 for the effect of length on the sulfonated ligand.

\section{Ex vivo activity}

In order to develop an effective antiviral strategy the active substances have to act mainly after infection. We verified whether MUS:OT-NPs were effective also after virus infection of cells. Cells were infected with wild-type HSV-2 (multiplicity of infection, MOI $0.01 \mathrm{pfu} / \mathrm{cell}$ ) for $2 \mathrm{~h}$ at $37^{\circ} \mathrm{C}$. After removal of the viral inoculum, different doses of MUS:OT-NPs were added to the cell monolayers immediately or after 2,4 or $24 \mathrm{~h}$. Cells and supernatants were harvested when the untreated wells exhibited a cytopathic effect of the whole monolayer. The cell free supernatants were then titrated. We determined that MUS:OT-NPs had an $\mathrm{EC}_{50}$ of $4.4 \mu \mathrm{g} / \mathrm{mL}$, with complete inhibition at $400 \mu \mathrm{g} / \mathrm{mL}$ and $3 \operatorname{logs}$ reduction at $80 \mu \mathrm{g} / \mathrm{mL}$ (Supplementary Figure 17) and a relevant inhibition of infection in all the tested time points. Thus the NPs can either prevent infection or block an ongoing infectious process depending on whether they inactivate the virus inoculum or the viral progeny.

To further verify the increased activity of our NPs in a model similar to an in vivo infection, we performed antiviral assays in EpiVaginal tissues. These are composed of human-derived ectocervical epithelial cells grown on a collagen-coated membrane to form a multilayered and highly differentiated tissue that is similar to the vaginal mucosa. We used HSV-2 as a challenge due to its specific tropism for the genital mucosa. HSV-2 $\left(10^{5} \mathrm{pfu}\right)$ was pre-incubated with the NPs at $500 \mathrm{nM}$ and then added on tissues for $2 \mathrm{~h}$ at $37^{\circ} \mathrm{C}$. The tissues were washed apically every day 
and the viral titer was evaluated by titration. The results show a significantly better profile of inhibition of the MUS:OT-NPs compared to the MES-NPs (Figure 4A). Moreover we performed experiments in EpiVaginal tissues also pre-treating the tissues for $18 \mathrm{~h}$ and subsequently infecting with HSV-2 or infecting the tissue with HSV-2 and treating 24 hpi. In both experiments we could observe a significant inhibition with MUS:OT-NPs (Figure $4 \mathrm{~B}$ and C) confirming the preventive and therapeutic activity of MUS:OT-NPs observed in cell lines. Moreover the nanoparticles proved to be non toxic in MTT and LDH assays conducted on EpiVaginal tissues (Supplementary Figure 18) demonstrating their biocompatibility with a human mucosa.

\section{In vivo activity}

To provide the proof of concept that MUS:OT-NPs could exert inhibitory activity also in vivo, we tested them in Balb/c mice infected with $\mathrm{RSV}^{43}$. Three groups of $5 \mathrm{BALB} / \mathrm{c}$ mice were treated at day 0 with (i) $50 \mu 1$ of PBS, (ii) $50 \mu 1$ of PBS, or (iii) MUS:OT-NPs in PBS (50 $\mu 1$ at 200 $\mu \mathrm{g} / \mathrm{ml}$ ) in the latter two cases this was followed, 10 minutes later, by inoculation with RSV-Luc $\left(10^{4} \mathrm{pfu}\right) .3$ days post-infection the luciferase expression in the lungs was analysed as a measure of the extent of infection. As shown in Figure 4D and 4E, untreated mice show a clear pulmonary dissemination of RSV infection. By contrast, the luciferase signal from the lungs of MUS:OT-NPs treated group was found to be statistically identical to the noise level set by the signal of uninfected mice treated solely with a PBS solution, indicating that MUS:OT-NPs treatment prevented the pulmonary dissemination of the infection.

Moreover, to investigate the biodistribution of MUS:OT-NPs, organ homogeneates were subjected to inductively coupled mass spectrometry (ICP-MS) where it was possible to detect gold presence only in lung homogenates while there was no detectable signal from spleen, liver and 
brain (Supplementary Figure 19). Of note, the localization on the MUS:OT-NPs is consistent with their antiviral activity in the lungs as shown in Figure 4D and 4E.

\section{Conclusions}

We believe that the approach presented here has a chance to produce medically relevant virucidal drugs to fight viral infections. See Supplementary Discussion 5 for the extension of this work to biodegradable nanoparticles. The results found so far show outstanding virucidal activity over HSV-2 and LS-VSV-G, while the activity versus HPV and RSV, although remarkable should be improved. In any case, it should be stressed that the strategy proposed is intrinsically broadspectrum, allowing the potential prevention and treatment of multiple viral infections with a single drug, a great advantage mostly in virology where rapid and at times unexpected infections occur. For example, West Nile, Yellow Fever, and Dengue are growing threats. All these viruses belong to the Flaviridae family, and are HSPG-binding viruses. Preliminary results with gold NPs show nanomolar virucidal efficacy over Dengue 2 (see Figure 1C and Supplementary Figure 20). Similarly, the Filoviridae family contains several human pathogens causing haemorrhagic fevers, including Ebola virus, for which drugs are urgently needed. All bind HSPGs as attachment receptors, and are potentially susceptible to the antiviral NPs presented in this study. Overall, what presented here is a first step towards the development of treatments (whether prophylactic or therapeutic will be determined by further in-depth in-vivo experimentations) for many worldwide threatening viral infections. 


\section{References:}

1 Lozano, R. et al. Global and regional mortality from 235 causes of death for 20 age groups in 1990 and 2010: a systematic analysis for the Global Burden of Disease Study 2010. Lancet (London, England) 380 , 2095--2128, doi:10.1016/S0140-6736(12)61728-0 (2012).

2 http://www.who.int/mediacentre/factsheets/fs310/en/.

3 Plotkin, S. A. Vaccines: past, present and future. Nature Medicine 11, S5--11, doi:10.1038/nm1209 (2005).

4 De Clercq, E. \& Li, G. Approved Antiviral Drugs over the Past 50 Years. Clinical Microbiology Reviews 29, 695--747, doi:10.1128/CMR.00102-15 (2016).

5 De Clercq, E. Strategies in the design of antiviral drugs. Nature Reviews. Drug Discovery 1, 13--25, doi:10.1038/nrd703 (2002).

6 Fridland, A., Connelly, M. C. \& Robbins, B. L. Cellular factors for resistance against antiretroviral agents. Antiviral Therapy 5, 181--185 (2000).

7 Spillmann, D. Heparan sulfate: Anchor for viral intruders? Biochimie 83, 811-817, doi:10.1016/s03009084(01)01290-1 (2001).

8 Cagno, V. et al. Highly sulfated K5 Escherichia coli polysaccharide derivatives inhibit respiratory syncytial virus infectivity in cell lines and human tracheal-bronchial histocultures. Antimicrobial Agents and Chemotherapy 58, 4782--4794, doi:10.1128/AAC.02594-14 (2014).

9 Lembo, D. et al. Auto-associative heparin nanoassemblies: a biomimetic platform against the heparan sulfate-dependent viruses HSV-1, HSV-2, HPV-16 and RSV. European Journal of Pharmaceutics and Biopharmaceutics: Official Journal of Arbeitsgemeinschaft Für Pharmazeutische Verfahrenstechnik e.V 88, 275--282, doi:10.1016/j.ejpb.2014.05.007 (2014).

10 Rusnati, M. et al. Sulfated K5 Escherichia coli polysaccharide derivatives: A novel class of candidate antiviral microbicides. Pharmacology \&\& Therapeutics 123, 310--322, doi:10.1016/j.pharmthera.2009.05.001 (2009).

11. Klimyte, E. M., Smith, S. E., Oreste, P., Lembo, D. \& Dutch, R. E. Inhibition of Human Metapneumovirus Binding to Heparan Sulfate Blocks Infection in Human Lung Cells and Airway Tissues. J. Virol. 90, 92379250 (2016).

12. Riblett, A. M. et al. A Haploid Genetic Screen Identifies Heparan Sulfate Proteoglycans Supporting Rift Valley Fever Virus Infection. J. Virol. 90, 1414-1423 (2015).

13. Donalisio, M. et al. The AGMA1 poly(amidoamine) inhibits the infectivity of herpes simplex virus in cell lines, in human cervicovaginal histocultures, and in vaginally infected mice. Biomaterials 85, 40-53 (2016).

14. Cagno, V. et al. The agmatine-containing poly(amidoamine) polymer AGMA1 binds cell surface heparan sulfates and prevents attachment of mucosal human papillomaviruses. Antimicrob. Agents Chemother. 59, 5250-5259 (2015).

15 Baram-Pinto, D., Shukla, S., Gedanken, A. \& Sarid, R. Inhibition of HSV-1 attachment, entry, and cell-tocell spread by functionalized multivalent gold nanoparticles. Small (Weinheim an Der Bergstrasse, Germany) 6, 1044--1050, doi:10.1002/smll.200902384 (2010). Bergstrom, D. E. et al. Polysulfonates derived from metal thiolate complexes as inhibitors of HIV-1 and various other enveloped viruses in vitro. Antiviral Chemistry $\mid \&$ Chemotherapy 13, 185--195 (2002).

17 Bowman, M.-C. et al. Inhibition of HIV fusion with multivalent gold nanoparticles. Journal of the American Chemical Society 130, 6896--6897, doi:10.1021/ja710321g (2008).

18 Scordi-Bello, I. A. et al. Candidate sulfonated and sulfated topical microbicides: Comparison of anti-human immunodeficiency virus activities and mechanisms of action. Antimicrobial Agents and Chemotherapy 49, 3607-3615, doi:10.1128/aac.49.9.3607-3615.2005 (2005).

19 McCormack, S. et al. PRO2000 vaginal gel for prevention of HIV-1 infection (Microbicides Development Programme 301): a phase 3, randomised, double-blind, parallel-group trial. Lancet (London, England) 376, 1329--1337, doi:10.1016/S0140-6736(10)61086-0 (2010).

20 Pirrone, V., Wigdahl, B. \& Krebs, F. C. The rise and fall of polyanionic inhibitors of the human immunodeficiency virus type 1. Antiviral Research 90, 168--182, doi:10.1016/j.antiviral.2011.03.176 (2011).

21 Van Damme, L. et al. Lack of effectiveness of cellulose sulfate gel for the prevention of vaginal HIV transmission. New England Journal of Medicine 359, 463-472, doi:10.1056/NEJMoa0707957 (2008). 
Shogan, B., Kruse, L., Mulamba, G. B., Hu, A. \& Coen, D. M. Virucidal activity of a GT-rich oligonucleotide against herpes simplex virus mediated by glycoprotein B. Journal of Virology 80, 47404747, doi:10.1128/jvi.80.10.4740-4747.2006 (2006). Bastian, A. R. et al. Cell-Free HIV-1 Virucidal Action by Modified Peptide Triazole Inhibitors of Env gp120. Chemmedchem 6, 1335-1339, doi:10.1002/cmdc.201100177 (2011). de Souza e Silva, J. M. et al. Viral Inhibition Mechanism Mediated by Surface-Modified Silica Nanoparticles. ACS Applied Materials \& Interfaces 8, 16564-16572, doi:DOI: 10.1021/acsami.6b03342 (2016). Bromberg, L. et al. Antiviral Properties of Polymeric Aziridine- and Biguanide-Modified Core-Shell Magnetic Nanoparticles. Langmuir 28, 4548-4558, doi:10.1021/la205127x (2012). Broglie, J. J. et al. Antiviral Activity of Gold/Copper Sulfide Core/Shell Nanoparticles against Human Norovirus Virus-Like Particles. PloS One 10, e0141050, doi:10.1371/journal.pone.0141050 (2015). Lara, H. H., Garza-Trevino, E. N., Ixtepan-Turrent, L. \& Singh, D. K. Silver nanoparticles are broadspectrum bactericidal and virucidal compounds. Journal of Nanobiotechnology 9, doi:10.1186/1477-31559-30 (2011).

Chen, N. N., Zheng, Y., Yin, J. J., Li, X. J. \& Zheng, C. L. Inhibitory effects of silver nanoparticles against adenovirus type 3 in vitro. Journal of Virological Methods 193, 470-477, doi:10.1016/j.jviromet.2013.07.020 (2013).

Abe, M. et al. Effects of several virucidal agents on inactivation of influenza, Newcastle disease, and avian infectious bronchitis viruses in the allantoic fluid of chicken eggs. Japanese Journal of Infectious Diseases 60, 342--346 (2007).

Chaudhuri, A., Battaglia, G. \& Golestanian, R. The effect of interactions on the cellular uptake of nanoparticles. Physical Biology 8, 046002, doi:10.1088/1478-3975/8/4/046002 (2011).

Lipowsky, R. \& Dobereiner, H. G. Vesicles in contact with nanoparticles and colloids. Europhysics Letters 43, 219-225, doi:10.1209/epl/i1998-00343-4 (1998).

Sabella, S. et al. A general mechanism for intracellular toxicity of metal-containing nanoparticles. Nanoscale 6, 7052--7061, doi:10.1039/c4nr01234h (2014).

Huang, R. X., Carney, R. P., Stellacci, F. \& Lau, B. L. T. Colloidal Stability of Self-Assembled Mono layer-Coated Gold Nanoparticles: The Effects of Surface Compositional and Structural Heterogeneity. Langmuir 29, 11560-11566, doi:10.1021/la4020674 (2013).

Huang, R. X., Carney, R. P., Stellacci, F. \& Lau, B. L. T. Protein-nanoparticle interactions: the effects of surface compositional and structural heterogeneity are scale dependent. Nanoscale 5, 6928-6935, doi:10.1039/c3nr02117c (2013).

Huang, R. X., Carney, R. R., Ikuma, K., Stellacci, F. \& Lau, B. L. T. Effects of Surface Compositional and Structural Heterogeneity on Nanoparticle-Protein Interactions: Different Protein Configurations. Acs Nano 8, 5402-5412, doi:10.1021/nn501203k (2014).

6 Bathia, S., Cuellar Camacho, L. \& Haag, R. Pathogen Inhibition by Multivalent Ligand Architectures. Journal of the American Chemical Society, doi:doi:10.1021/jacs.5b12950 (2016). Dasgupta, J. et al. Structural Basis of Oligosaccharide Receptor Recognition by Human Papillomavirus. Journal of Biological Chemistry 286, 2617-2624, doi:10.1074/jbc.M110.160184 (2011).

8 Knappe, M. et al. Surface-exposed amino acid residues of HPV16 11 protein mediating interaction with cell surface heparan sulfate. Journal of Biological Chemistry 282, 27913-27922, doi:10.1074/jbc.M705127200 (2007).

Qian EQ, Wixtrom AI, Axtell JC, Saebi A, Rehak P, Han Y, Moully EH, Mosallaei D, Chow S, Messina M, Wang JY, Royappa AT, Rheingold AL, Maynard HD, Kral P, Spokoyny AM. Atomically Precise Organomimetic Cluster Nanomolecules Assembled via Perfluoroaryl-Thiol SNAr Chemistry. Nature Chem. 9, 333-340 (2016).

Matulis, D. \& Lovrien, R. 1-Anilino-8-naphthalene sulfonate anion-protein binding depends primarily on ion pair formation. Biophys. J. 74, 422-429 (1998).

Melcrova, A. et al. The complex nature of calcium cation interactions with phospholipid bilayers. ScientificReports 6, 38035 (2016).

Rameix-Welti, M.-A. et al. Visualizing the replication of respiratory syncytial virus in cells and in living mice. Nat Commun 5, 5104 (2014). 
Acknowledgments: F.S. and his laboratory were supported in part by the Swiss National Science Foundation NRP 64 grant, and by the NCCR on bio-inspired materials. D.L. was supported by a grant from University of Turin (ex 60\%). J.H. and J.W. were supported by research grant from the Ministry of Education, Youth and Sports of the Czech Republic (LK11207). C.T., L.K. and F.S. were supported by Leenards Foundation. P.K. was supported by the NSF DMR-1506886 grant. L.V. was supported by the startup funding from UTEP. M.G. and R.L. thank the MIMA2 platform for access to the IVIS-200, which was financed by the Ile de France region (SESAME). M.M. thanks Ricardo C Guerrero-Ferreira for the tomograms aquisition.

\section{Author contributions:}

V.C. was responsible for all activities involving HSV2, HPV, RSV under the supervision of D.L. and EpiVaginal experiments under the supervision of C.T. and L.K. P.A. and M.D. were responsible for all testing with VSV-LV-G under the direction of S.K.. P.J.S. was responsible for NP and ligand synthesis. M.M. was responsible for all cryo-TEM. S.T.J. was responsible for Iron oxide NP synthesis. M.G. R.L. were responsible for the in vivo experiments, R.W.M. and J.F.E. engineered the RSV-Luc used for in vivo experiments. M.V. was responsible for stained TEM imaging of the viruses. J.H. and J.W conducted all testing with DENV-2. S.S. and Y.H. were responsible for molecular dynamics simulations under the direction of P.K. and L.V.E.R.J. synthesised MUP-NPs. A.B. synthesised MES NPs. B.S. synthesised EG2OH-NPs. C.M. and P.A. conducted the gel electrophoresis. M.D. was responsible for HSV-1 and 2 and dose response experiments. F.S. and S.K. first conceived the experiments, F.S. and D.L. developed the interpretation of the experiments. F.S., D.L., V.C., and S.T.J. wrote the paper.

\section{Competing Financial Interests}

The authors have no competing Financial Interest.

\section{Data Availability Statement}

Raw data of experiments are available at https://figshare.com/s/19ed37fbbe0261a00254

\section{Methods:}

Detailed procedures are provided in the Methods section in the Supplementary Information

Nanoparticles synthesis

MUS:OT and all MUS nanoparticles were synthesised using a slightly modified procedure reported by Verma et al. ${ }^{45}$ all MES Au nanoparticles were synthesised following the synthetic procedure reported by Baram-Pinto et al. ${ }^{15}$

Viral inhibiton

Viruses were pre-incubated with nanoparticles for $1 \mathrm{~h}$ and then added on cells. Viral infection was evaluated through plaque assay or FACS.

$\underline{\text { Virucidal assays }}$ 
Viruses $\left(10^{4}-10^{6} \mathrm{pfu}\right)$ and 100 to $1000 \mu \mathrm{g} / \mathrm{ml}$ of MUS:OT-NPs were incubated at different time points at $37^{\circ} \mathrm{C}$ and the virucidal effect was investigated with serial dilutions of the mixtures. Viral titers were calculated at dilutions at which the NPs were not effective.

\section{TEM and Cryo TEM}

HSV-2 and HPV-PsV ( $\left.10^{5} \mathrm{pfu}\right)$ were incubated with or without $100 \mu \mathrm{g} / \mathrm{ml}$ Au-NPs and were adsorbed on carbon- and Formvar-coated grids and negatively stained with $0.5 \%$ uranyl acetate and observed with CM 10 electron microscope. For cryo TEM, viruses and NPs were flashfrozen in their native hydrated state on carbon coated grids and imaged at $-175^{\circ} \mathrm{C}$ in a FEI Tecnai F20 Cryo 200kV TEM.

\section{Ex vivo analysis}

Epivaginal tissues were purchased from Mattek and cultured as indicated by the manufacturer. The tissues were infected with HSV-2 $\left(10^{4}-10^{5} \mathrm{pfu}\right)$ and treated with different NPs (500-1500 $\mathrm{nM}$ ) and titrations of supernatants were conducted on Vero cells to evaluate viral replication.

$\underline{\text { In vivo analysis }}$

Balb/c mice were treated with $200 \mu \mathrm{g} / \mathrm{ml}$ of MUS:OT-NPs and then infected with RSV-Luc as previously described ${ }^{43}$. Luminescence was measured using the IVIS 200 imaging system (Xenogen Corp.).

\section{$\underline{\text { Statistics }}$}

All results are presented as the mean values and sem from three independent experiments. The $\mathrm{EC}_{50}$ values for inhibition curves were calculated by regression analysis with GraphPad Prism. The selectivity indexes SI were calculated dividing the $\mathrm{CC}_{50}$ for the $\mathrm{EC}_{50}$. A unpaired t-test analysis was performed for virucidal assays and in vivo assays. $t$ values and degrees of freedom (df) are indicated in figure legends. 


\section{FIGURE}

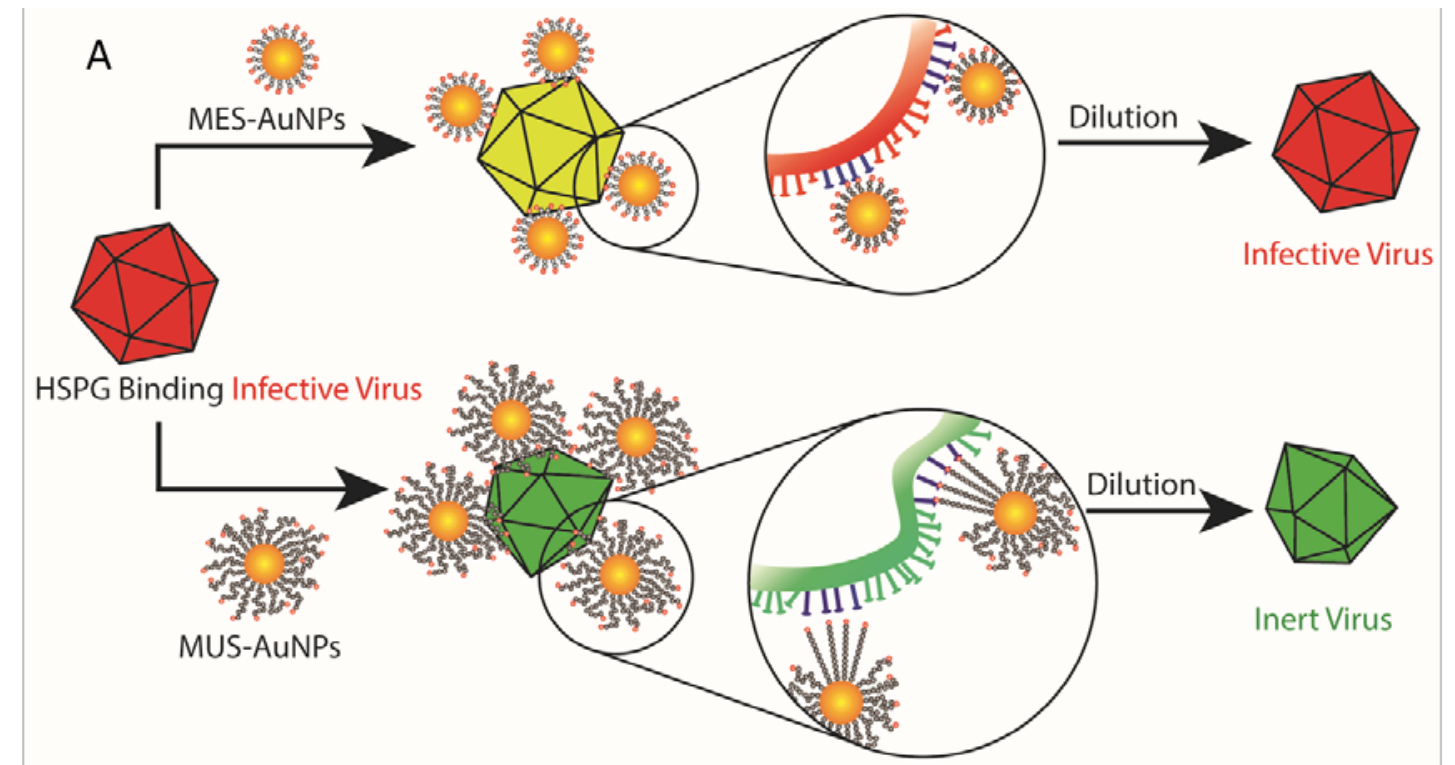

B
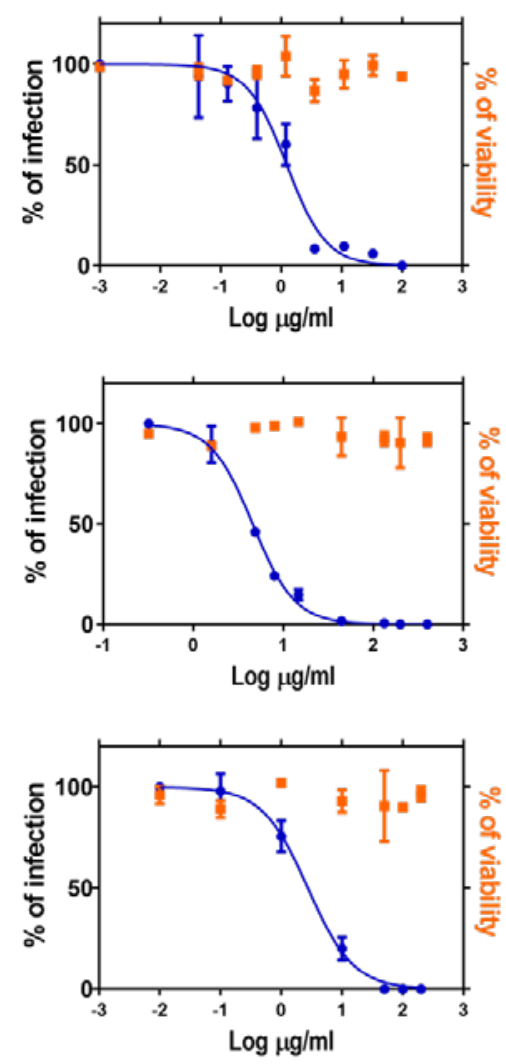

C
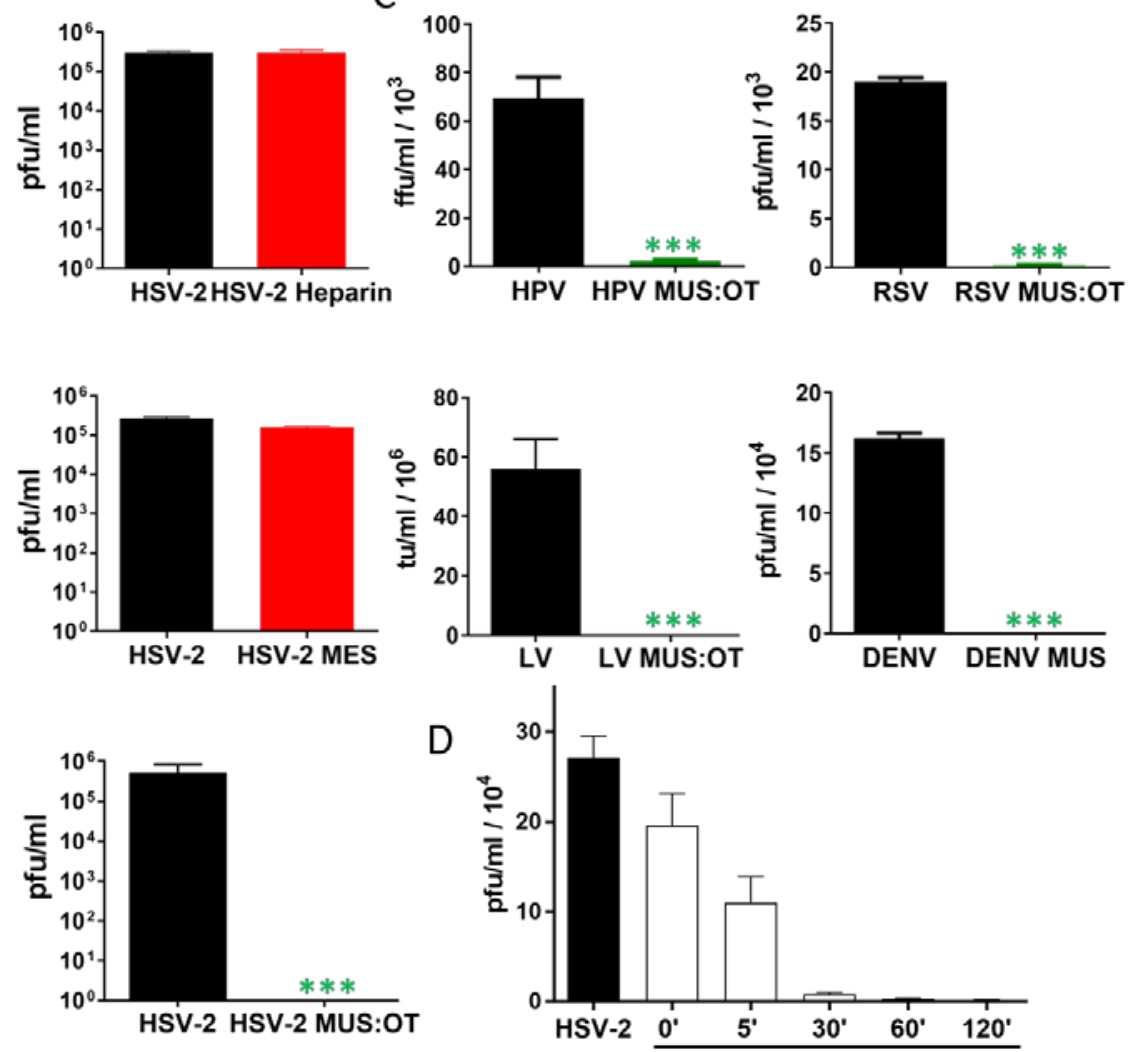

Figure 1. Virucidal activity of MUS:OT-NPs. A) Cartoon of the virucidal activity of MUS:OTNPs compared to MES-NPs B) From top to bottom Heparin, MES-NPs and MUS:OT-NPs viral infectivity curves and virucidal assays. The percentages of infection were calculated comparing the number of plaques in treated and untreated wells. C) Virucidal activity of MUS coated NPs 
against HPV-16, RSV, LV-VSV-G (indicated as LV), and DENV-2 viruses. D) MUS:OT-NPs inhibition of viral infectivity against HSV-2 versus time (minutes). Results are the mean and sem of 3 independent experiments performed in triplicate. ${ }^{* *} \mathrm{p}<0.001$ (two-tailed) in unpaired $\mathrm{t}$ test analysis. HSV-2 $\mathrm{t}=0.9788 \mathrm{df}=17$, HPV $\mathrm{t}=7.776 \mathrm{df}=16$, RSV $\mathrm{t}=44.32 \mathrm{df}=6, \mathrm{LV} \mathrm{t}=5.6 \mathrm{df}=2$, DENV $\mathrm{t}=38, \mathrm{df}=4$. 


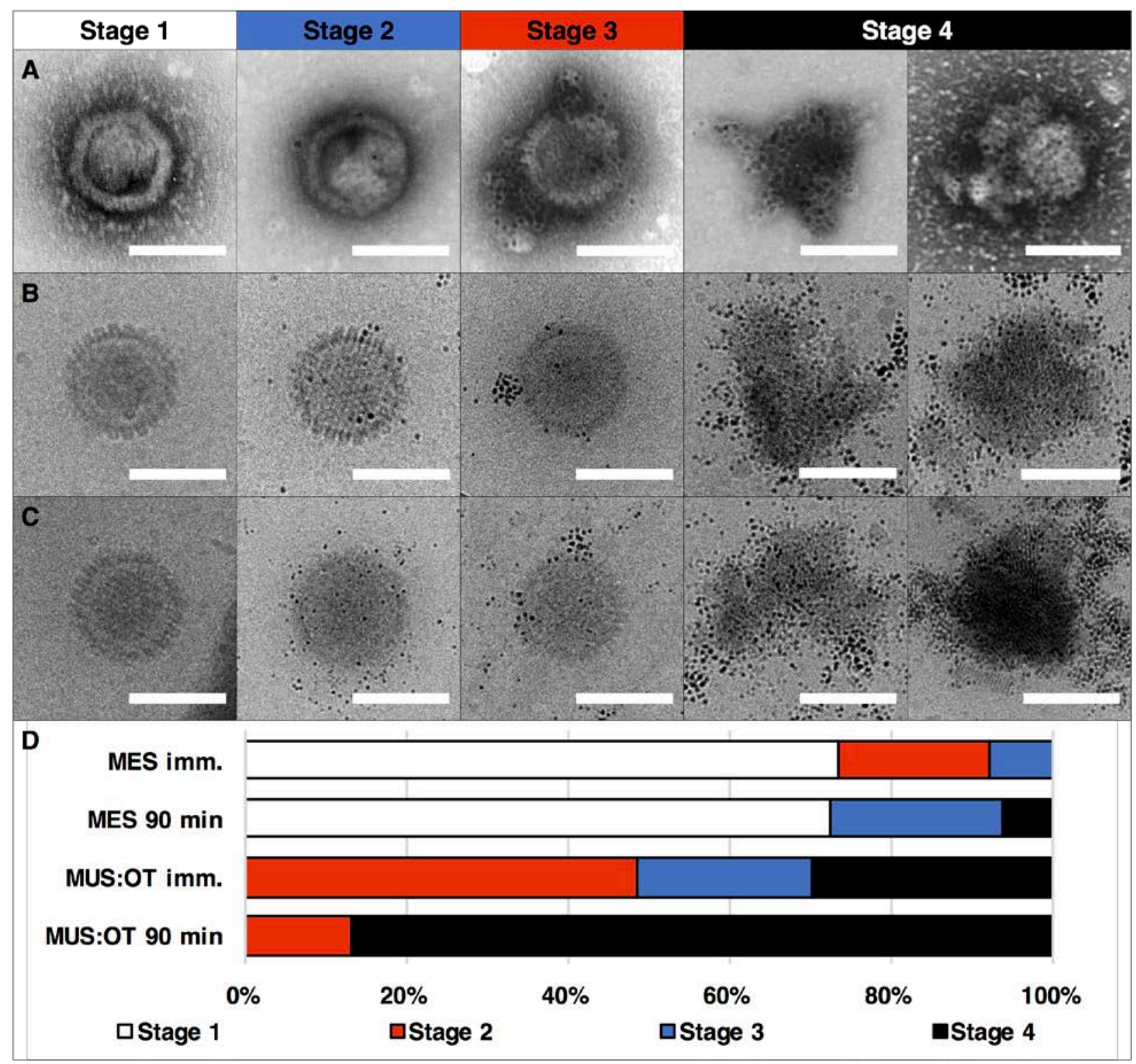

Figure 2: HSV-2 and its association with MUS:OT-NPs. The samples were imaged using dry negatively stained TEM (A) or unstained cryo-TEM (B,C). The scale bars are $100 \mathrm{~nm}$. D) Percentage and distribution of NPs (MES or MUS:OT) associated with HSV-2 immediately and after 90 min were determined by analysing between 50 and 100 cryo-TEM images per condition. 


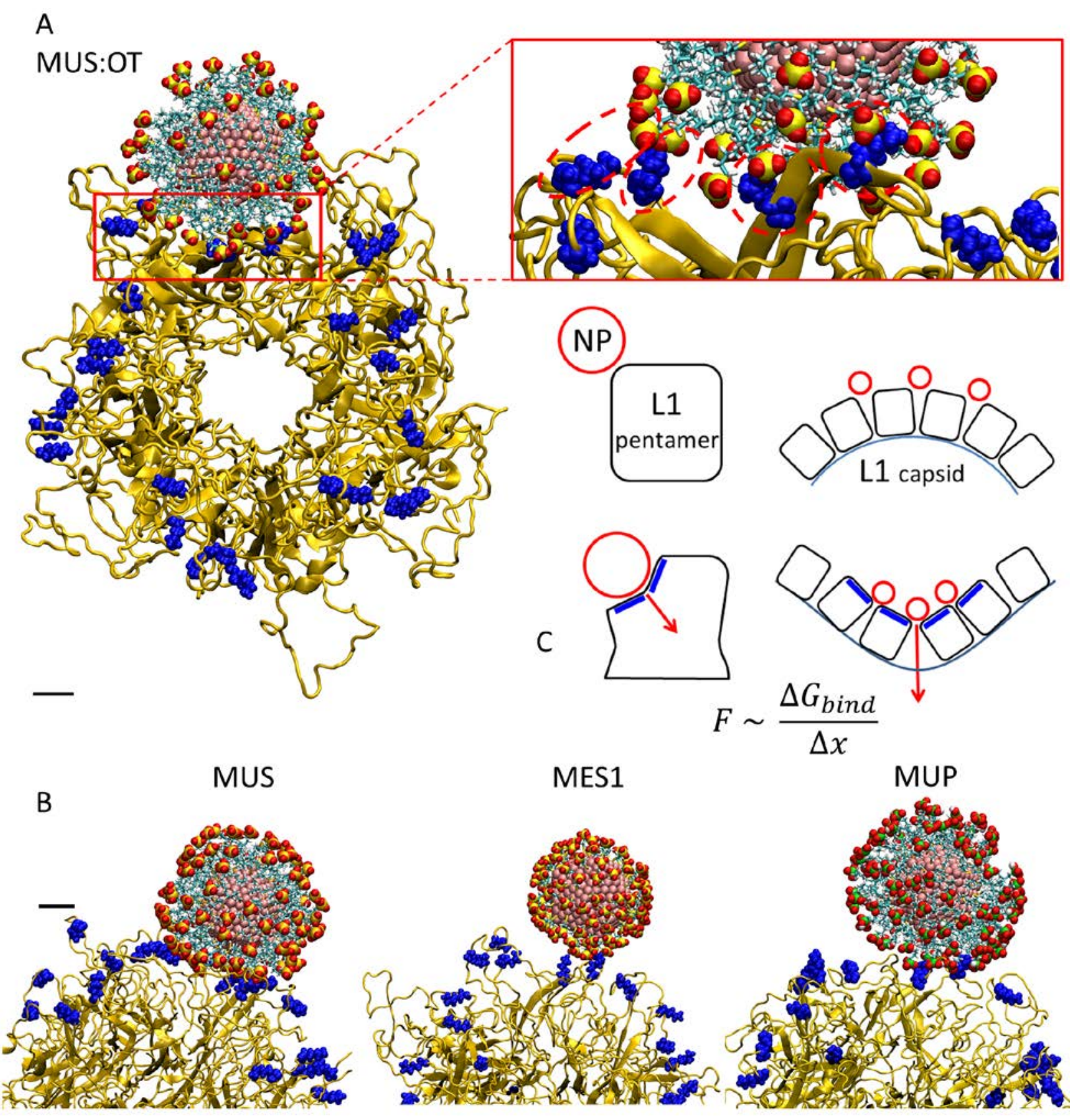

Figure 3: Molecular Dynamics Simulations. A) Top view of a small sulfonated MUS:OT-NP (2.4 nm core) selectively binding to HPV capsid L1 protein pentamer, after $25 \mathrm{~ns}$ of simulations. Red and yellow spheres show negatively charged terminal sulfonate groups of the MUS-NP. Positively charged HSPG-binding residues of L1 (K278, K356, K361, K54 and K59) are shown in blue. Inset highlights the strong selective coupling between sulfonate groups and HSPG-binding residues (K356, K361, K54 and K59). B) Side view of the interactions of MUS, MES1 (Figure 
S20 shows $5 \mathrm{~nm}$ MES2) and MUP NPs with a HPV L1 protein pentamer. Strong multivalent binding is developed within $15 \mathrm{~ns}$ simulation only for MUS NP. C) Schematic diagram illustrates how strong multi-site binding of MUS-type NPs to HSPG-binding residues can induce irreversible changes in the arrangement of L1 capsid proteins. Scale bars are $1 \mathrm{~nm}$. 

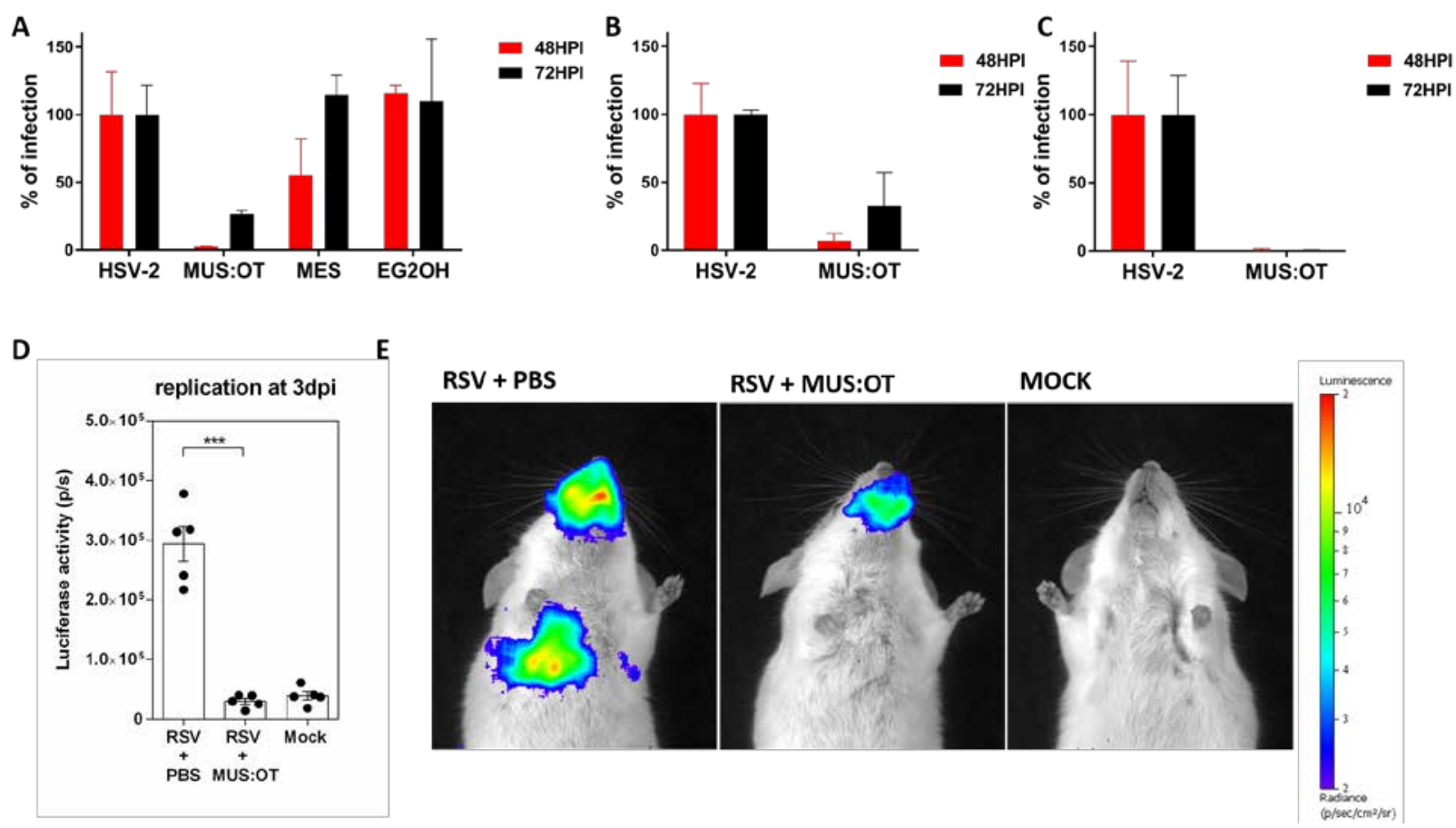

Figure 4: MUS:OT-NPs activity ex vivo and in vivo. A) NPs activity against HSV-2 infected human cervicovaginal histocultures with pre-incubation of virus and NPs (500 nm) and addition on tissues. B) EpiVaginal tissues were treated with MUS:OT-NPs for $18 \mathrm{~h}$ and subsequently infected. C) EpiVaginal tissues were infected with HSV-2 and after 24 h MUS:OT-NPs were added on tissues. The percentages of infection were calculated comparing the viral titers in the treated tissues supernatant and in the untreated. Results are the mean and sem of 3 independent experiments performed in triplicate. D) Groups of $5 \mathrm{BALB} / \mathrm{c}$ mice were treated at day 0 with either $50 \mu \mathrm{l}$ of PBS or MUS:OT-NPs in PBS (50 $\mu \mathrm{l}$ at $200 \mu \mathrm{g} / \mathrm{ml})$ and 10 minutes later where inoculated with RSV-Luc, with the exception of the mock group. Bioluminescence was measured at day 3 post-infection by intranasal injection of D-luciferin. Capture of photon emission was performed using the IVIS system. Luciferase activities were quantified for each mouse using Living Image software. Luciferase activity is expressed as photons per second (p/s). Results are the mean and sem of 3 independent experiments performed with 5 mice per group. $* * * p<0.001$ (two-tailed) in unpaired $\mathrm{t}$ test analysis. $\mathrm{t}=8.976 \mathrm{df}=8 \mathrm{E}$ ) Ventral views of representative mice. The scale on the right indicates the average radiance: the sum of the photons per second from each pixel inside the region of interest/number of pixels $(\mathrm{p} / \mathrm{s} / \mathrm{cm} 2 / \mathrm{sr})$. 